\title{
Gwendolyn Macewen: versiones de su poesía ${ }^{1}$
}

\section{Gwendolyn Macewen: versions of her poetry}

\author{
Aimée G. Bolaños ${ }^{2}$ \\ Hugh Hazelton ${ }^{3}$
}

Submetido em 2 e aprovado em 13 de novembro de 2017.

Resumen: La traducción al español de seis poemas de Gwendolyn MacEwen (Toronto 1941-1987), al completarse treinta años de su muerte y la publicación del último libro de sus poemas, Afterworlds, motivan este trabajo conjunto que incluye una presentación de la poeta para el lector latinoamericano, así como un comentario sobre los referidos poemas y la tarea traductora. Aspiramos a compartir una experiencia de lectura pensando que la traducción literaria implica un proceso creativo cultural en su sentido más amplio $\mathrm{y}$, en este caso, de inmersión en el universo de la autora, especialmente de su poética.

Palabras claves: Poesía canadiense contemporánea. Gwendolyn MacEwen. Poética. Traducción de poemas.

\begin{abstract}
This year, which marks the thirtieth anniversary of Gwendolyn MacEwen's death (Toronto 1941-1987), as well as of the publication of Afterworlds, her last book of poems, is a fitting moment for a joint collaboration to present her work to a Latin American audience, along with a commented translation of six of her poems. We wish to share a reading experience based on the idea of literary translation as a creative cultural process in the widest sense, including an immersion in the author's universe, particularly in regard to her poetics.
\end{abstract}

Keywords: Contemporary Canadian poetry. Gwendolyn MacEwen. Poetics. Translation of poetry.

\section{La poeta en su espejo}

Do not think for one minute it is the Poem that matters. Is not the Poem that matters.

You can shove the Poem.

What matters is what is out there in the large dark and in the long light, Breathing.

De Afterworlds

Entre las figuras cimeras de la literatura canadiense contemporánea, Gwendolyn MacEwen (1941-1987) es ave rara. De intensa y corta vida, para algunos de sus estudiosos 
vivida en justa medida, completa en sí, aunque mucho más hubiera podido ser realizado, su personalidad creadora marca a sus contemporáneos y encuentra lectores apasionados. Nacida en un Toronto poco cosmopolita en esos años y precedida por una generación de poetas mujeres que se mantenían dentro de moldes convencionales, su escritura irrumpe con la fuerza de la diferencia, inicialmente influenciada por la contracultura Beat, sobre todo en el apelo a la espiritualidad oriental.

Margaret Atwood, excelente conocedora de la obra de MacEwen desde la propia condición de escritora, deja un vívido testimonio de la poeta leyendo su poesía en los años 60 , era de mitologías, utopías y cambios sociales radicales. Con su largo cabello, rica voz y apariencia juvenil unida a la autoridad poética, sus oyentes podían tener la sensación de estar compartiendo un delicioso secreto (ATWOOD, 1993, p. IX). Misterio y placer que también estarán presentes en los acercamientos ulteriores a su poesía.

Mayormente y siempre poeta, también escribe novelas de vidas imaginarias, (Julian, the Magician, 1963; King of Egypt, King of Dreams, 1971), narrativa corta (Noman's Land, 1985), dramas, memorias de viaje (Mermaids and Ikons: A Greek Summer, 1978) y literatura para niños. Además, traduce. Compone poemas desde los diez años, publica con dieciséis. Entre 1961 y 1987 realiza una obra de gran envergadura. En esa poesía, amplia y diversa, se destacan The Drunken Clock (1961), The Rising Fire (1963), A Breakfast for Barbarians (1966), The Shadow-Maker (1969), The Armies of the Moon (1872), The T. E. Lawrence Poems (1983) y Afterworlds (1987).

Estudia de modo autodidacta varias lenguas -hebreo, árabe, griego, francés-, viaja hacia diversos espacios socio-culturales (Israel, Medio Oriente, Egipto, Grecia). Transita del taoísmo al gnosticismo y la cábala. Va al encuentro de las dimensiones visionarias y míticas de los pueblos originarios de Canadá. Se sumerge en los saberes de la historia árabe antigua y la egiptología, muy atraída por los jeroglíficos. Su biblioteca imaginaria es inclusiva. Heterodoxa, bebe de diferentes fuentes, sin dejar de ser ella. Si algo puede caracterizarla es la pasión cognoscitiva unida a la búsqueda metafísica. Fascinada por el misterio de la existencia, explora lo arcano, se abre a sabidurías ocultas, penetra en conciencias y arquetipos, persigue la iluminación mística. Se interroga e interroga al mundo, adentrándose en el espacio sin fin de la espiritualidad. Disonante y solitaria, 
escribe vuelta hacia sí, distante de las convenciones de la institución literaria. Existe para y en la escritura. Como autora busca una comunicación fundada en las afinidades electivas, expresiva en hondura.

De la extensa obra de Gwendolyn MacEwen, escasamente traducida al español, hemos escogidos seis poemas representativos de su arte en una trayectoria no exactamente cronológica, sino más bien de poética y cosmovisiva. Son ellos: "Meditation of a Seamstress" (1) (The Armies of de Moon), "Water" (The T. E. Lawrence Poems), "Poems in Braille" (A Breakfast for Barbarians), "Dark Pines under Water" (The ShadowMaker), "The Shadow-Maker" (The Shadow-Maker) y "The Garden of the Thieves" (Afterworlds). Los poemas también fueron elegidos, y no en segundo lugar, por motivos personales, imantados por su plenitud estética y por profundas resonancias al ficcionalizar una experiencia de vida y creación artísticas. Aun así, y tomando sus palabras, vale decir que lo verdaderamente importante no es el poema, sino lo que está más allá de él, vivo.

\title{
EI espejo de los poemas
}

\begin{abstract}
Poetry has got nothing to do with poetry. Poetry is how the air goes green before thunder, is the sound you make when you come, and why you live and how you bleed, and the sound you make or don't make when you die.
\end{abstract}

De Afterworlds

En "Meditation of a Seamstress" (1), la autora se autorretrata con la forma simbólicometafórica de una costurera desesperada y alucinada trabajando en una obra inexplicable, urgida por el tiempo y lo caótico del intento para finalmente consubstanciarse con la hierba. Con esa poderosa imagen, Gwendolyn MacEwen se presenta de una forma tan nítida como ambigua, tal es la riqueza contradictoria de su poesía al categorizar el mundo y a sí misma, viendo en enigma desde su condición de mujer artista. Algo vital está en juego, acaso la Puntada Perdida de esa costurera al borde del abismo que medita sobre el sentido de la existencia, mientras vuelve a un estado natural primigenio, transfigurada y haciendo. 
Creadora de mundos reales, íntimamente vinculados entre sí, que pueden tener forma fantástica, onírica, mítica, mágica, su poesía fluye de un libro a otro, de un poema a otro, para configurarse en la afirmación y negación. Así la metáfora del agua -río de Heráclito en perpetuo devenir- es perfecta para caracterizar su poética. En "Water", el elemento vital del agua contiene la infinitud. Posee todos los colores, sin embargo carece de color, tal es su poder de metamorfosis. Encuentra siempre el camino de regreso a casa. Es todo: principio y final, hasta historia.

A semejanza del agua, la poética de MacEwen acoge lo elemental y esencial en movimiento transformador. Mudan las formas, mas se mantiene fiel a sí misma. Sus textos ofrecen diferentes imágenes personales y transpersonales, imposibles de separar en el intrincado tejido de identidad-alteridad que la distingue.

En este juego identitario, la figura autoral se proyecta en la musa que, a la vez, la redimensiona como autora. En este imaginario de la identidad, la musa usualmente aparece como "principio complementario, una figura que la guía hacia la desconocida oscuridad, obligándola a confrontarse con el cambio y el misterio, en una lucha de contrarios" (POTVIN, 1999, p. 33). Subvirtiendo los roles habituales de la historia de la poesía, la mujer artista no solo da vida a la musa, ahora masculina, sino toma su identidad, convirtiéndola de objeto de inspiración en sujeto de la enunciación, confundidos rostros y voces en la reafirmación de su poder creador.

En este sentido, T. E. Lawrence pudiera ser una de sus más intensas representaciones metafóricas por su carga de contradicciones como viajero colonial de dimensiones épicas y antiheróicas; buscador insaciable, perdido de sí, expresivo del “deseo y la imposibilidad de armonizar las diferencias" (SHOSTAK, 2001, p. 25). Lawrence deviene máscara, figura icónica del imaginario, ideal y conflictiva. Travestida, transgresora, sin límites, Gwendolyn MacEwen transborda de sí, a semejanza del agua del poema, tan patente en todo su imaginario.

Desde muy temprano, la poeta se vuelca a las palabras como materia prima del oficio, atenta a sus vínculos con el mundo de la vida. En el espíritu del surrealismo, de marcada presencia en su poética, le concede existencia a las palabras que se convierten en lo que nombran y traen a la vida lo nombrado. Sus palabras son demiurgos. En "A 
Breakfast for Barbarians" el yo poético con brazos y piernas hace alfabetos, tiene manosverbos. Al nominar, toca el mundo. El poema descubre en la familiaridad, la distancia para testimoniar artísticamente un proceso de extrañamiento que ya ha sido dominante en las vanguardias, sobre todo surrealista y expresionista. El conocimiento brota de esa distancia desfamiliarizadora, de esa percepción de lo esencial con ojos naturales, despojados.

De modo emblemático, la autora toma como referencia la cultura de los escribas del antiguo Egipto. El jeroglífico del ojo -tanto icónico como alegórico- se transforma en ojo real para volver al tejido de la ficción, simbolizando un proceso típico de la poesía de MacEwen que subvierte temporalidades y borra las fronteras de realidad y ficción, apariencia y esencia, sueño y vigilia.

La palabra poética permite descubrir en otra escala de pensamiento, con frecuencia paradójico. En la estela homérica, la poeta "ve" desde la ceguera, lee en braille con el tacto de sensibilidad aguzada, abriéndose al tercer ojo de la espiritualidad hasta depararse con el misterio inefable del jeroglífico final, quién sabe si referido al par dialéctico de vida-muerte. Lejos del hermetismo, su palabra es lúcida y traslúcida. Tanto transcendente como coloquial, esotérica y del cotidiano. Alusiva y elusiva.

En "Dark Pines under Water", la deslumbrante metáfora de los pinos-memoria posee una compleja significación relativa al misticismo telúrico de la memoria colectiva, patente en la poesía canadiense que acoge la tradición visionaria de los primeros pueblos de la región, a la vez que de finos tintes subjetivos individuales. Con sus pinos sumergidos en el lago furtivo del adentro, la autora se interpreta en versión dialógica, conectándose con esa dimensión mística originaria.

Sabedora de que hay más cuartos dentro que en el exterior, como ya ha dicho en una cita memorable, busca en el espejo de las aguas la imagen interior, angustioso sueño y gracia poderosa. Su descenso órfico es revelador: una exploración de sí que lleva a una epifanía, no por eso menos dolorosa. Ahondando cada vez más al navegar su profundidad ignota, aparece algo elemental y durmiente que debe ser dicho, que la poeta quiere decir. La visión, siendo tan intensamente del yo, resulta transnarcisista: el sujeto poético se reconoce y figura en la pulsión creativa al traspasar su oscuridad vital. 
En ese universo ambivalente de luces y sombras como polaridades complementarias, concepción milenaria que se remota al Tao, "The Shadow-Maker" pudiera ser leído a modo de luminoso poema de amor -al otro, a sí mismo-, interpenetrados amante y amado en la busca del Uno místico, compartiendo, no sin lucha, la original oscuridad, augurio de luz. El poema crea un mítico hacedor de sombra y, a la par, la poeta se reconoce su criatura como figura abismal del poema. Sin la necesaria sombra, que se inscribe en su carne y le da corporeidad, sería apenas infinita curva estéril, onda de luz ruinosa en el aire.

Un mítico jardín que existe en la memoria perdida de la infancia es la metáfora seminal que sustenta "The Garden of Thieves". El yo enunciativo cuenta una historia que se abre a múltiples sentidos, si bien coloca en un primer plano a la mujer que escribe, figura de la ficción, que durante años ha querido escribir un poema a partir de ese título intrigante. En el sueño aparecen claves, y la soñadora recupera el jardín-paraíso perdido, pero ha sido invadido por ladrones que le roban el Gran Poema, también "Gran Paradoja” (WOOD, 2000, p. 56) de la escritura imposible-posible, significativamente ya escrito por Anonymus.

Gwendolyn MacEwen invoca un motivo secular que recorre la historia de la poesía: el poeta no existe, es su obra. Retomando de manera implícita la figura paradigmática de Homero - magistralmente imaginado por Jorge Luis Borges en "El Hacedor" desde esta perspectiva-, el hacedor de poemas se ha diluido en su sombra, y sus contornos se esfuman en el absoluto de la poesía, patrimonio de todos. El poema nos deja con una imagen de gran belleza imaginal, la mujer que sueña la poesía continúa su búsqueda antes de que la noche caiga.

Leída como espiritualista, realista mágica, surrealista, gótica, mística, fetichista, new age, feminista avant la lettre, revisitando la historia del arte y la mitología, visionaria y de la experiencia, su poesía se resiste a clasificaciones e interpretaciones cerradas $^{4}$. Perteneciente a una genealogía artística en la que el escritor es una figura engendrada por la propia ficción -Lord Byron, una referencia fundamental en la modernidad-, la poeta va por la vida con sus atuendos estéticos fabulosos, se funde con sus diseños, delirante y clarividente -imposible no pensar en William Blake-, entre mitomanía y autoconfesión, revelándose y ocultándose en la autoficción y las vidas imaginarias. A veces, casi visible; otras, mítica, fantástica. De cualquier modo envuelta en el claroscuro de la sombra. 
Al autoficcionalizarse, más cercana a Proteo que a Narciso, las formas de identidad son mutantes. En sus metamorfosis se evidencia la compleja problemática del ser: artista, mujer, persona. La lectura de sus textos es un juego de espejos, también laberinto, donde al perdernos, estamos en camino de encontrarnos. Ciertamente su propuesta estética fascina a lectores de cualquier temporalidad y cultura, tornándolos actores de la ficción poética.

Desafiadora y transgresiva, para Gwendolyn MacEwen la poesía nada tiene que hacer con la poesía, es como se vive. Con marcas de época y fuera del tiempo, signo de la poesía verdadera, más allá de tendencias y modismos, marginal, pero en el centro del espíritu estético de la alta modernidad posvanguardista, su peculiar visión artística le permite no solo la participación apasionada en su tiempo de escritura, sino crear sentidos que, inagotables, transcienden. Leída hoy, su obra fulgura.

Para terminar, un breve comentario sobre este ejercicio. Entendida la traducción como un proceso dinámico cognitivo, hemos pretendido darle vida a un nuevo evento de lenguaje, al interpretar formas y sentidos tan elusivos en la poesía. Como es sabido, la gama de significados de la tarea traductora es amplia: trasladar, trasmutar, recrear, crear, tal vez todos y ninguno definitorio. Difícil y tentadora nos ha resultado la tarea de leer signos polisémicos en el intento de otra enunciación. Siendo así, dejamos constancia de que ni en todos los casos quedamos felices o, al menos, satisfechos con nuestras elecciones, alternando hallazgos y desconciertos al mediar entre visiones, culturas, lenguas, sensibilidades, aunque como la edición es bilingüe, el lector podrá comparar y ensayar sus interpretaciones. En verdad, la traducción es escritura que implica la lectura crítica. La mejor traducción debe ser la mejor lectura.

Leer a Gwendolyn MacEwen ha sido un placer laberíntico. Sus palabras continuamente dicen lo que dicen y mucho más que no puede ser verbalizado, pero está poéticamente presente. Los poemas se abren a sentidos insólitos que la lengua no consigue traducir, si bien quedan alentando, respirando, en busca de esos lectores-autores, traductores, que con su carga vivencial sabrán darle los más insospechados alcances. Cada poema, que a continuación dejamos para la lectura, es un espejo de aguas, de sombras luminosas, en el que podemos reconocernos y ser tocados por la gracia de la poesía. 


\section{Poemas de Gwendolyn MacEwen}

\section{MEDITATIONS OF A SEAMSTRESS (1)}

From: The Armies of the Moon. Toronto: Macmillan, 1972.

When it's all too much to handle

and the green seams of the world start fraying,

I drink white wine and sew

like it was going out of style;

curtains become dresses, dresses

become pillowcases, clothes

I've worn forever get taken in or out.

Now I can't explain exactly

what comes over me, but when the phone rings

I tell people I'm indisposed;

I refuse to answer the door, I even

neglect my mail.

(Something vital is at stake

the Lost Stitch or the Ultimate Armhole,

I don't know what) and hour after hour

on the venerabe Singer

I make strong strong seams for the dresses

and my world.

The wine possesses me

and I sew like a fiend, forgetting to use

the right colours of thread, unable to make

a single straight line;

I know somehow I'm fighting time

and if it's not all done by nightfall

everything will come apart again;

continental shelves will slowly drift into the sea

and earthquakes will tear wide open

the worn-out patches of Asia.

Dusk, a dark needle, stabs the city

and I get visions of chasing fiery spools of thread

mile after mile over highways and fields

until I inhabit some place at the hem of the world

where all the long blue draperies

of skies and rivers wind;

spiders' webs describe

the circling of their frail thoughts forever;

everything fits at last and someone has lined

the thin fabric of this life I wear with grass. 


\section{MEDITACIONES DE UNA COSTURERA (1)}

Cuando es más de lo que puedo soportar

y las costuras verdes del mundo comienzan a deshilacharse, tomo vino blanco y coso

como si estuviera pasando de moda; cortinas se vuelven vestidos, vestidos se vuelven fundas de almohada, ropa que he usado siempre se achica o agranda. Ahora no puedo explicar exactamente lo que me pasa, pero cuando suena el teléfono le digo a la gente que estoy indispuesta; me niego a abrir la puerta, incluso descuido mi correo.

(Algo vital está en juego la Puntada Perdida o la Última Sisa, qué sé yo) y hora tras hora en la Singer venerable hago fuertes fuertes costuras para mis vestidos y mi mundo.

El vino me posee y coso como un demonio, olvidándome de usar los hilos de colores adecuados, incapaz de hacer una sola línea recta; yo sé que de alguna manera lucho contra el tiempo y si todo no está listo antes del anochecer se va a deshacer otra vez; plataformas continentales caerán lentamente en el mar y los terremotos rasgarán de par en par los gastados remiendos de Asia.

El crepúsculo, una aguja oscura, apuñala la ciudad $\mathrm{y}$ tengo visiones de perseguir bobinas de hilo en llamas milla tras milla en carreteras y campos hasta que habite algún lugar en el dobladillo del mundo donde todas las largas cortinas azules de cielos y ríos se enrollan; las telarañas describen las vueltas de sus pensamientos frágiles para siempre; todo encaja al fin y alguien ha forrado el tejido fino de esta vida que llevo con hierba. 


\section{WATER}

From: The T. E. Lawrence Poems. Toronto: Mosaic Press, 1983.

When you think of it, water is everything. Or rather, Water ventures into everything and becomes everything. It has

All tastes and moods imaginable; water is history

And the end of the word is water also.

I have tasted water

From London to Miranshah. In France it tasted

Of Crusaders' breastplates, swords, and tunnels of rings

On ladies' fingers.

In the springs of Lebanon water had

No colour, and was therefore all colours, outside of Damascus

It disguised itself as snow and let itself be chopped

And spooned onto the stunned red grapes of summer.

For years I have defended water, even though I am told there are other drinks.

Water will never lie to you, even when it insinuates itself Into someone else's territory. Water has style.

Water has no conscience and no shame; water thrives on water, is self-quenching.

It often tastes of brine and ammonia, and always

Knows its way back home.

When you want to travel very far, do as the Bedouin doDrink to overflowing when you can, and then

Go sparingly between wells.

\section{AGUA}

Si lo piensas, el agua es todo. O mejor, El agua se aventura en todo y se hace todo.

Tiene

Todos los gustos y humores imaginables; el agua es historia Y el fin del mundo es agua también.

He probado el agua

Desde Londres hasta Miranshah. En Francia tenía el gusto de los petos y espadas de los cruzados y de túneles de anillos Sobre los dedos de las damas.

En los manantiales de Líbano el agua no 
Tenía color, y por tanto era de todos los colores, fuera de Damasco

Se disfrazaba de nieve y se dejó ser cortada

Y servida en cucharadas sobre las atónitas uvas rojas de verano.

Por años he defendido el agua, aunque me dicen

que hay otras bebidas.

El agua nunca te mentirá, aun cuando se insinúe

En el territorio de otro. El agua tiene estilo.

El agua no tiene conciencia ni vergüenza; el agua

se crece con agua, apaga su propia sed.

A menudo sabe al mar y a amoniaco, y siempre

Conoce el camino de vuelta a casa.

Cuando quieras viajar muy lejos, haz como los beduinos:

Bebe hasta desbordar cuando puedas

y luego

Ve con moderación entre los pozos.

\section{POEMS IN BRAILLE}

From: A Breakfast for Barbarians. Toronto: The Ryerson Press, 1966.

1

all your hands are verbs,

now you touch worlds and feel their names -

thru the thing to the name

not the other way thru (in winter

I am Midas, I name gold)

the chair and table and book

extend from your fingers;

all your movements

command these things back to their

places; a fight against familiarity

makes me resume my distance

2

they knew what it meant, those egyptian scribes who drew eyes right into their hieroglyphs, you read them dispassionate until the eye stumbles upon itself blinking back from the papyrus 
outside, the articulate wind annotates this; I read carefully lest I go blind in both eyes, reading with that other eye the final hieroglyph

\section{3}

the shortest distance between 2 points on a revolving circumference is a curved line; O let me follow you, Wencelas

\section{4}

with legs and arms I make alphabets like in those children's books where people bend into letters and signs, yet I do not read the long cabbala of my bones truthfully; I need only to move to alter the design

5

I name all things in my room and they rehearse their names, gather in groups, form tesseracts, discussing their names among themselves

I will not say the cast is less than the print I will not say the curve is longer than the line, I should read all things like braille in this season with my fingers I should read them lest I go blind in both eyes reading with that other eye the final hieroglyph

\section{POEMAS EN BRAILLE}

1

todas tus manos son verbos, ahora tocas mundos y sientes sus nombres a través de la cosa al nombre no por otro camino (en el invierno soy Midas, nombro el oro)

la silla y la mesa y el libro se extienden de sus dedos; todos tus movimientos mandan estas cosas a volver a su lugar; una lucha contra la familiaridad me hace recuperar mi distancia 
2

sabían lo que significaba,

esos escribas egipcios que dibujaban

los ojos directamente dentro de sus jeroglíficos,

los lees desapasionada hasta que

el ojo tropieza con sí mismo

parpadeando en respuesta desde el papiro

afuera, el viento elocuente

lo anota; leo cuidadosamente

para que no me quede ciega de ambos ojos, al leer con

el otro ojo el último jeroglífico

3

la distancia más corta entre 2 puntos

sobre una circunferencia giratoria

es una línea curva; $O$ déjeme seguirte,

Wenceslao

4

con piernas y brazos hago alfabetos

como en esos libros infantiles

donde la gente se curva sobre letras y signos,

sin embargo no leo la larga cábala de mis huesos

sinceramente; solo necesito moverme para cambiar el diseño

5

nombro todas las cosas en mi cuarto

y ellas ensayan sus nombres,

se reúnen en grupos, forman teseractos,

discutiendo sus nombres entre ellas

no diré que el molde es menos que la impresión

no diré que la curva es más larga que la línea,

debería leer todas las cosas como el braille en esta época

con los dedos las debería leer

para que no me quede ciega de ambos ojos al leer con

el otro ojo el último jeroglífico

\section{DARK PINES UNDER WATER}

From: The Shadow-Maker. Toronto: Macmillan, 1969.

This land like a mirror turns you inward

And you become a forest in a furtive lake;

The dark pines of your mind reach downward, 
You dream in the green of your time, Your memory is a row of sinking pines.

Explorer, you tell yourself, this is not what you came for Although it is good here, and green;

You had meant to move with a kind of largeness,

You had planned a heavy grace, an anguished dream.

But the dark pines of your mind dip deeper

And you are sinking, sinking, sleeper

In an elementary world;

There is something down there and you want it told.

\section{PINOS OSCUROS BAJO EL AGUA}

Esta tierra como un espejo te vuelve hacia adentro

$\mathrm{Y}$ te conviertes en un bosque dentro de un lago furtivo;

Los pinos oscuros de tu mente van hacia abajo,

Sueñas con el verde de tu época,

Tu memoria es una fila de pinos hundidos.

Explorador, te dices, no es por eso que llegaste

Aunque sea bueno aquí, y verde;

Querías moverte con una especie de amplitud,

Habías previsto una pesada gracia, un sueño angustiado.

Pero los pinos oscuros de tu mente se sumergen más profundo

Y te hundes, te hundes, durmiente

En un mundo elemental;

Hay algo allá abajo y quieres decirlo.

\section{THE SHADOW-MAKER}

From: The Shadow-Maker. Toronto: Macmillan, 1972.

I have come to possess your darkness, only this.

My legs surround your black, wrestle it

As the flames of day wrestle night

And everywhere you paint the necessary shadows

On my flesh and darken the fibres of my nerve;

Without these shadows I would be

In air one wave of ruinous light

And night with many mouths would close

Around my infinite and sterile curve.

Shadow-maker create me everywhere

Dark spaces (your face is my chosen abyss), 
For I said I have come to possess your darkness, Only this.

\section{EL HACEDOR DE SOMBRAS}

He llegado para poseer tu oscuridad, solo eso. Mis piernas rodean tu negrura, luchan con ella Como las llamas del día luchan con la noche $\mathrm{Y}$ en todas partes pintas las sombras necesarias Sobre mi carne y oscureces las fibras de mi nervio;

Sin estas sombras sería

En el aire una onda de luz ruinosa

Y la noche con muchas bocas se cerraría

Alrededor de mi curva infinita y estéril.

Hacedor de sombras créame por todos lados

Espacios oscuros (tu cara es mi abismo escogido), Porque dije que he llegado para poseer tu oscuridad, Solo eso.

\section{THE GARDEN OF THE THIEVES}

From: Afterworlds. McClelland \& Stewart, 1987.

For years I have wanted to write a poem called The Garden of the Thieves.

The title turns up in old notebooks with asterisks Surrounding it and arrows pointing to it, and Notes telling me to write it, write it, but

It never got written until now because I never knew where the garden was, or who

The thieves were, so the naked title lay there Between sheets of paper that seethed with reason And grand ideas, until one night I actually dreamed

Of the garden where I played as a child, and it was invaded by thieves

Who stole the Great Poem from me, the one we all know

Never gets written, and I saw the title as they

Whisked it away, and yes, it was beyond a doubt

The Garden of the Thieves, written by Anonymous who was my favourite poet And who I thought was a Byzantine king. I have been Pondering over this for quite some time, and thought I'd better get it all down before the night falls. 


\section{EL JARDÍN DE LOS LADRONES}

Por muchos años he querido escribir un poema llamado

El jardín de los ladrones.

El título aparece en viejos cuadernos rodeado de asteriscos y

Con flechas que lo señalan, $\mathrm{y}$

Notas diciéndome que lo escriba, lo escriba, pero

Nunca se ha escrito hasta ahora porque nunca supe

dónde estaba el jardín, ni quiénes

Eran los ladrones, así que el título desnudo quedaba ahí

Entre hojas de papel que se agitaban con razón

Y grandes ideas, hasta que una noche realmente soñé

Con el jardín donde jugaba cuando niña, y fue

invadido por ladrones

Que me robaron el Gran Poema, el que todos sabemos

Nunca se escribe, y vi el título mientras

Me lo quitaron, y sí, no había duda de que era

El jardín de los ladrones, escrito por Anónimo

que era mi poeta preferido

Y que pensaba era un rey bizantino. Medito

Sobre eso desde hace mucho tiempo, y pensé

Que debería ponerlo todo por escrito antes que anochezca.

\section{Referencias}

ATWOOD, Margaret. MacEwen's Muse. Second Words. Toronto: Anansi, 1982.

. Introduction: The Early Years. In: Gwendolyn MacEwen. Volume One. The Early Years. Margaret Atwood; Barry Callaghan, Ed. Toronto: Exile Editions, 1993, p. VII-XII.

DAVEY, Frank. Gwendolyn MacEwen: The Secret of Alchemy. Surviving the Paraphrase. Winnipeg: Turnstone, 1983.

GERRY, Thomas M.F. 'Green Yet Free of Seasons': Gwendolyn MacEwen and the Mystical Tradition of Canadian Poetry. In: Studies in Canadian Literature 16:2 (1991): 147-61.

HARDING-RUSELL, Gilliam. Gwendolyn MacEwen's 'The Nine Arcana of the Kings' as Creative Myth and Paradigm. In: English Studies in Canada 15:2 (1988): 204-217.

. Iconic Mythopoeia in MacEwen's The T.E. Lawrence Poems. In: Studies in

Canadian Literature 9:1 (1984): 95-107.

MACEWEN, Gwendolyn. The Drunken Clock. Toronto: Aleph Press, 1961 . The Rising Fire. Toronto: Contact, 1963.

. A Breakfast for Barbarians. Toronto: Ryerson, 1966. 
. The Shadow-Maker. Toronto: Macmillan, 1969.

. The Armies of the Moon. Toronto: Macmillan, 1972.

. Mermaids and Icons: A Greek Sumner. Toronto: Anansi Press, 1978.

. The T. E. Lawrence Poems. Toronto: Mosaic Press, 1983.

. Noman's Land. Toronto: Coach House, 1985.

. Afterworlds. Toronto: McClelland and Stewart, 1987.

POTVIN, Liza. Gwendolyn MacEwen and Female Spiritual Desire. In: Canadian Poetry:

Studies, Documents, Reviews 28 (Spring/Summer 1991) 18-39.

SHOSTAK, Dorothy. Open Secrets: Fetishcity in the Poetry of Gwendolyn MacEwen. Dalhousie University, Halifax, 2001. [Tesis doctoral].

STRIMAS, Meaghan. The Selected Gwendolyn MacEwen. Introduction by Rosemary Sullivan. Holstein: Exile Editions, 2007.

SULLIVAN, Rosemary. Shadow Maker: The Life of Gwendolyn MacEwen. Toronto: HarperCollins, 1995.

WARWICK, Ellen D. To Seek a Single Symmetry. In: Canadian Literature (1977) 21-34.

WOOD, Brent. From The Rising Fire to Afterworlds: the Visionary Circle in the Poetry of Gwendolyn MacEwen. In: Canadian Poetry 47 (2000): 40-69.

\section{Notes}

1 Artículo concebido y realizado conjuntamente, con atención especial de Hugh Hazelton en la traducción y de Aimée G. Bolaños en el texto de presentación.

2 Profesora del Programa de Posgraduación en Letras, Universidade Federal do Rio Grande (FURG), Brasil. Profesora adjunta de la Universtity of Ottawa, Canadá. aimee@vetorial.net.

3 Profesor emérito de Español, Concordia University, Montreal, Quebec, Canada.

4 Con esta notable variedad de interpretaciones, especialmente resultan aportadores los estudios críticos de Margaret Atwood, Margaret Sullivan, Brent Woods, Dorothy Shostak, Frank Davies, Liza Potvin, Elle Warwick, Thomas Gerry y Gilliam Harding-Rusell. Ver: Referencias. 